\title{
$0.9 \%$ Sodium chloride solution versus Plasma-Lyte 148 versus compound sodium lacTate Solution in children admitted to PICU - a randomised controlled trial - SPLYT-P: study protocol for an intravenous fluid therapy trial
}

Sainath Raman ( $\nabla$ Sainath.raman@uq.edu.au )

The University of Queensland https://orcid.org/0000-0002-0152-9980

Andreas Schibler

The University of Queensland

Renate Le Marsney

The University of Queensland

Peter Trnka

The University of Queensland

Melanie Kennedy

The University of Queensland

Adrian Mattke

The University of Queensland

Kristen Gibbons

The University of Queensland

Luregn Schlapbach

University Children Hospital Zurich: Universitats-Kinderspital Zurich

\section{Study protocol}

Keywords: Balanced solutions, bolus, child, critical care, intravenous fluid therapy

Posted Date: April 8th, 2021

DOI: https://doi.org/10.21203/rs.3.rs-390577/v1

License: (c) (i) This work is licensed under a Creative Commons Attribution 4.0 International License.

Read Full License 


\section{Abstract}

Background: Intravenous fluid therapy represents the most common intervention critically ill patients are exposed to. Hyperchloremia and metabolic acidosis associated with $0.9 \%$ sodium chloride have been observed to lead to worse outcomes, including mortality. Balanced solutions, such as PlasmaLyte-148 and Compound Sodium Lactate, represent potential alternatives but the evidence on optimal fluid choices in critically ill children remains scarce. The aim of this study is to demonstrate whether balanced solutions, when used as the intravenous fluid therapy, are able to reduce the incidence of a rise in serum chloride level compared to $0.9 \%$ sodium chloride in critically ill children.

Methods: This is a single centre, open label randomised controlled trial with parallel 1:1:1 assignment into three groups: $0.9 \%$ sodium chloride, Plasma-Lyte 148 , and Compound Sodium Lactate solutions for intravenous fluid therapy. The intervention includes both maintenance and bolus fluid therapy. Children age $<16$ years admitted to intensive care and receiving intravenous fluid therapy during the first four hours of admission are eligible. The primary outcome measure is a ${ }^{3} 5 \mathrm{mmol} / \mathrm{L}$ increase in serum chloride level within 48 hours post randomisation. The enrolment target is 480 patients. The main analyses will be intention-to-treat.

Discussion: This study tests three types of intravenous fluid therapy in order to compare the risk of hyperchloremia associated with normal saline versus balanced solutions. The pragmatic study is thereby assessing the most common intervention in paediatric critical care.

This is a single centre open label study with no blinding at the level of delivery of intervention. Certain Paediatric Intensive Care Unit (PICU) patient groups such as patients admitted with a cardiac condition or following traumatic brain injury are excluded from this study.

Trial registration: The study has received ethical approval (HREC/19/QCHQ/53177: 06/06/2019). It is registered in the Australian New Zealand Clinical Trials Registry (ACTRN12619001244190) from $9^{\text {th }}$ September 2019. Recruitment commenced on $12^{\text {th }}$ November 2019. The primary results manuscript will be published in a peer reviewed journal.

\section{Administrative Information}

\section{Administrative information}

Note: the numbers in curly brackets in this protocol refer to SPIRIT checklist item numbers. The order of the items has been modified to group similar items (see http://www.equator-network.org/reportingguidelines/spirit-2013-statement-defining-standard-protocol-items-for-clinical-trials/). 
Title $\{1\}$

$0.9 \%$ Sodium chloride solution versus Plasma-Lyte 148 versus compound sodium lacTate Solution in children admitted to PICU - a randomised controlled trial SPLYT-P

Trial registration $\{2 a$ and $2 b$.

Protocol version $\{3\}$

Funding $\{4\}$
The trial is registered in the Australian New Zealand Clinical Trials Registry (ACTRN12619001244190).
Protocol Version 7, dated 29/07/2020

The study was supported by a grant from the Study, Education and Research Trust Account funding scheme 2019, Children's Health Queensland, Brisbane, Australia. The funding sources had no involvement in study design, analyses, nor interpretation of the results.
Author details $\{5 a\}$

\section{Dr Sainath Raman ${ }^{1,2}, \mathrm{MRCPCH}, \mathrm{PhD}, \mathrm{FCICM}$}

2. Prof. Dr. Andreas Schibler ${ }^{1,2}, \mathrm{MD}, \mathrm{FCICM}$

3. Renate Le Marsney ${ }^{1}$, BMedSc, MPH

4. Assoc. Prof. Dr. Peter Trnka ${ }^{3}$, MD, FRACP, MSc

5. Melanie Kennedy ${ }^{1,2}$, BNursing

6. Assoc. Prof. Dr. Adrian Mattke ${ }^{1,2}$ MBBS, MD, FRACP, FCICM

7. Assoc. Prof. Dr. Kristen Gibbons ${ }^{1}$, BlnfoTech, BMaths (Hons), PhD

8. Assoc. Prof. Dr. Luregn J Schlapbach ${ }^{1,2,4}$, MD, PhD, FCICM

1. Paediatric Critical Care Research Group, Child Health Research Centre, The University of Queensland, Queensland, Australia

2. Paediatric Intensive Care Unit, Queensland Children's Hospital, South Brisbane, Australia.

3. Paediatric Nephrology, Queensland Children's Hospital, South Brisbane, Australia

4. Pediatric and Neonatal Intensive Care Unit, Children`s Research Center, University Children's Hospital Zurich, Zurich, Switzerland

Name and contact information for the trial sponsor $\{5 b\}$
Ms. Jen Spodziak

Research Partnership manager

Faculty of Medicine

The University of Queensland, 
288 Herston Rd, Herston, QId 4006 Australia. SPIRIT guidance: Name and contact information for the trial sponsor.

T: +61733465358

Role of

sponsor $\{5 c\}$

The sponsor (The University of Queensland) and the funding body (Children's Health Queensland) had no involvement in study design, analyses, nor interpretation of the results.

\section{Introduction}

\section{Background and rationale $\{6 a\}$}

Intravenous fluid therapy (IVFT) is one of the most common interventions in critically ill patients [1]. Intravenous fluid therapy captures both maintenance as well as bolus fluid administration, and volumes of $100 \mathrm{ml} / \mathrm{kg}$ and more in the first days of paediatric intensive care unit (PICU) admission have been reported [2]. $0.9 \%$ sodium chloride (NS), available since the $18^{\text {th }}$ century [3], represents the default fluid for IVFT in many services worldwide [4-6]. However, administration of NS has been associated with hyperchloremia and metabolic acidosis [7, 8]. These effects of NS are attributed to its high chloride content with zero strong-ion difference [9]. Compound Sodium Lactate (CSL), containing lower sodium and chloride content, has been available and in use for nearly a century [3]. More recently, Plasma-Lyte $148(\mathrm{PL})$ has become available as an alternative balanced fluid with a higher sodium concentration as compared to CSL [10] .

Several interventional studies in adult critically ill patients have investigated if balanced IVFT solutions lead to improved clinical outcomes [1]. Two recent trials showed a significant reduction in need for renal replacement therapy favouring CSL compared to NS [11, 12]. Another randomised controlled trial (RCT), comparing NS to PL demonstrated no difference in the incidence of new onset acute kidney injury (AKI) between the groups [13]. A large RCT is underway comparing PL to NS used for both resuscitation and maintenance therapy in critically ill adult patients (NCT02721654).

The evidence to support optimal fluid choice in critically ill children is scarce and there is a lack of larger trials assessing fluid type in a broad PICU population. A matched retrospective cohort study comparing NS and CSL in patients less than 18 years of age with severe sepsis or septic shock showed no difference in mortality, incidence of acute kidney injury (AKI), new dialysis or length of stay [14]. Kartha et al., in a randomised study, demonstrated similar clinical and biochemical outcomes between CSL and NS in children with acute diarrhoeal illness [15]. Allen et al. observed greater increase in serum bicarbonate and more rapid improvement in dehydration scores [16] with CSL compared to NS. Balanced solution in perioperative IVFT in children undergoing brain tumor resection was associated with lower incidence of chloride increase [17]. Balamuth et al. recently reported on a pilot feasibility RCT in children with sepsis that comparing CSL and NS, but the study was not powered for clinical outcomes [18]. While results are awaited from a large RCT in India enrolling 710 children with sepsis comparing balanced solutions to normal saline (NCT02835157), another multi-centre RCT in children with sepsis has started recruiting 
(NCT04102371). While all these trials are directed at providing high level evidence for the use of balanced solutions versus $0.9 \%$ sodium chloride, they are restricted to specific disease groups and may not be representative of the wider PICU population.

The aim of this study is to demonstrate if, in critically ill children admitted to PICU, balanced solutions (PL and CSL), when compared to $0.9 \%$ sodium chloride as the IVFT, reduce the incidence of rise in serum chloride level in the first 48 hours post-PICU admission.

\section{Objectives $\{7\}$}

1. To determine if balanced solutions cause less chloride rise compared to $0.9 \%$ sodium chloride as IVFT in critically ill children in PICU.

2. To determine if balanced solutions cause less renal dysfunction compared to $0.9 \%$ sodium chloride as IVFT in critically ill children in PICU.

3. To conduct an IVFT trial in children to inform the design of a larger multicentre study powered for patient-centred primary outcome.

\section{Trial design $\{8\}$}

Single centre, open-label three-arm RCT of children aged birth to $<16$ years of age admitted to PICU and treated with IVFT.

\section{Methods: Participants, Interventions And Outcomes}

\section{Study setting $\{9\}$}

Paediatric Intensive Care Unit in Queensland Children's Hospital, Brisbane, Australia.

\section{Eligibility criteria $\{10\}$}

Eligible children are identified on admission to the mixed PICU (Table 1). Included are neonates, infants and children $<16$ years of age requiring IVFT within the past 4hours, which must be within the first 24hours of PICU admission. The exclusion criteria are patients who require disease-specific protocols for IVFT, certain pre-existing conditions such as cardiac patients, major electrolyte abnormalities, and futility. Cardiac patients are excluded because of the local practice of using minimal maintenance fluids and using Albumin $4 \%$ as the main bolus fluid type in this patient group. Patients with known pre-existing renal disease are not eligible for recruitment as some of the secondary outcomes specifically investigate the impact of the intervention on renal function. This includes patients admitted to PICU post-renal transplantation.

\section{Table 1: Inclusion and exclusion criteria}




\begin{tabular}{|c|c|c|}
\hline Group & Criterion & Definition \\
\hline \multicolumn{3}{|c|}{ Inclusion (determined on admission to PICU) } \\
\hline & Age & Birth to $<16$ years of age \\
\hline & Location & Admitted to PICU \\
\hline & Time frame & $\begin{array}{l}\text { New admission to PICU within the last } 24 \text { hours and has } \\
\text { received IVFT for } \leq 4 \text { hours in PICU }\end{array}$ \\
\hline & $\begin{array}{l}\text { Decision - Clinician } \\
\text { decides that IVFT is } \\
\text { required }\end{array}$ & $\begin{array}{l}\text { IVFT - All intravenous fluids (boluses and maintenance fluids } \\
\text { but not drug dilutions) administered in PICU }\end{array}$ \\
\hline & Biochemistry & $\begin{array}{l}\text { Admission sodium }>130 \mathrm{mmol} / \mathrm{L} \text { (measured on admission or } \\
\text { no longer than } 48 \text { hours before randomisation) }\end{array}$ \\
\hline \multicolumn{3}{|c|}{ Exclusion } \\
\hline & Age & $\geq 16$ years \\
\hline & Time frame & Received IVFT for $>4$ hours in PICU \\
\hline & \multirow[t]{2}{*}{ Pre-existing conditions } & - Admitted for a cardiac condition \\
\hline & & - Chronic kidney disease \\
\hline & \multirow{6}{*}{$\begin{array}{l}\text { Disease specific IVFT } \\
\text { protocols }\end{array}$} & - Traumatic brain injury or at risk of cerebral edema \\
\hline & & - Burns \\
\hline & & - Post-liver transplant \\
\hline & & - Post-renal transplant \\
\hline & & - Diabetic Ketoacidosis \\
\hline & & - Oncology patients needing hyperhydration \\
\hline
\end{tabular}

\section{Who will take informed consent? $\{26 a\}$}

Where possible, prospective informed consent will then be sought from parents by the research nurse or doctor. Due to the emergency nature of admissions to PICU, it is anticipated that in certain situations timely informed consent may not be feasible. In these cases, 'consent to continue' will be employed. The consent process should be completed by 72 hours after randomisation. If consent is received after 72 hours, this will be recorded as a protocol deviation.

\section{Additional consent provisions for collection and use of participant data and biological specimens \{26b\}}

Not applicable. No other biological specimens will be collected for storage.

\section{Interventions}




\section{Explanation for the choice of comparators $\{6 \mathrm{~b}\}$}

The three arms of the trial are:

1. Control arm - $0.9 \%$ Sodium Chloride solution ("normal saline", NS)

2. Intervention arm 1 - Plasma-Lyte 148 (PL)

3. Intervention arm 2 - Compound Sodium Lactate (CSL)

\section{Intervention description \{11a\}}

From the time of randomisation onwards, the IVFT of the enrolled patient must be provided as per the allocated arm. IVFT includes both maintenance fluids and any potential bolus fluids during the stay in PICU. Total parenteral nutrition will not be considered IVFT. Drug dilutions are not considered IVFT for the study purpose. If a patient was already on another fluid at time of randomisation, the protocol requires the IVFT to change to the allocated fluid. If clinicians decide to provide another study fluid, or another fluid (such as Albumin 4\%), the event will be considered a protocol violation and captured as such.

Dose and duration of IVFT: Decisions on indication, dose and duration of IVFT are made by the treating clinician. This ensures a pragmatic approach. The decision to start and stop maintenance and bolus IVFT is also made by the treating clinician.

Other PICU care: An arterial, venous, or capillary blood gas with measurement of $\mathrm{pH}$, sodium and chloride will be performed at the time of randomisation (if no lab results or blood gas are available in the previous 12 hours) and at least every 24 hours thereafter until discharge while the patient is on IVFT and in PICU. All other treatments such as electrolyte supplementation, medications, dextrose infusion, colloid and blood product administration are as per the local institutional procedures. Routine laboratory monitoring of children receiving IVFT such as daily blood tests to assess organ dysfunction are as directed by the clinician.

\section{Criteria for discontinuing or modifying allocated interventions $\{11 \mathrm{~b}\}$}

Discontinuation of intervention fluid will be at the discretion of the attending clinician. The potential reasons for this might be a new change in clinical status that makes the patient meet one of the exclusion criteria or study closure by the site. Participants' clinical data as per the case report form, with parent's permission, will be used for data analysis even after cessation of intervention. If the serum sodium drops to $128 \mathrm{mmol} / \mathrm{L}$, the patient will exit the study.

\section{Strategies to improve adherence to interventions $\{11 \mathrm{c}\}$}

Clinicians and bedside nurse will have ongoing education to improve adherence to the protocols.

\section{Relevant concomitant care permitted or prohibited during the trial $\{11 \mathrm{~d}\}$}


Total parenteral nutrition, enteral feeds and fluids, fluid used in drug dilution, fluid used as intravenous flush and blood products (packed red cells, fresh frozen plasma, cryoprecipitate) will not be considered for protocol violations.

\section{Provisions for post-trial care $\{30\}$}

Patients will exit study on discharge from PICU. There is not further follow-up planned.

\section{Outcomes $\{12\}$}

The primary outcome is a dichotomous variable defined as an increase in serum chloride ${ }^{3} 5 \mathrm{mmol} / \mathrm{L}$ within 48 hours from the time of randomisation. Secondary clinical outcomes include organ dysfunction free survival, new onset AKI, length of PICU stay, length of hospital stay, and PICU free survival (Table 2). New onset AKI will be additionally calculated using predicted baseline values where a baseline serum creatine value has not been measured [19-21]. Secondary safety outcomes focus on adverse events with respect to electrolyte derangements and are censored at 48 hours from the time of randomisation.

\section{Rationale for the primary outcome}

In a retrospective cohort study in 1935 children admitted to a mixed tertiary PICU, Barhight et al observed a 2.3 times increased odds of death (95\% confidence interval: 1.03 to 5.21) associated with an increase in chloride level of ${ }^{3} 5 \mathrm{mmol} / \mathrm{L}$ in the first 24 hours after PICU admission [22]. The increase in chloride value was defined as the difference between the admission (first level obtained after PICU admission) and maximum chloride level obtained during the first calender day [22]. Correspondingly, in a post-hoc analysis of the Fluid Expansion of Supportive Therapy Study, Levin et al. demonstrated a $1.65 \mathrm{mmol} / \mathrm{L}$ ( $95 \%-\mathrm{Cl} 0.47$ to 2.83 ) mean change in serum chloride level in children who received a $10 \mathrm{ml} / \mathrm{kg}$ bolus of NS or $4 \%$ albumin relative to those who did not receive a fluid bolus and this was associated with mortality [23]. Therefore, we have chosen a cutoff of ${ }^{3} 5 \mathrm{mmol} / \mathrm{L}$ increase in chloride level as our primary outcome.

Table 2: Definition of outcome measures 


Outcome Criterion Definition

\section{Primary}

Hyperchloremia Increase in serum chloride $5 \mathrm{mmol} / \mathrm{L}$

- Difference from baseline level to the highest chloride level measured within 48 hours post randomisation

- Baseline level: measured on blood gas at randomisation or using the closest lab or blood gas value up to a maximum of 12 hours before randomisation

- Highest chloride level: obtained in the first 48 hours post randomisation

- Patients will be assumed to have no hyperchloremia if chloride has not been measured in the first 48 hours post randomisation

\section{Secondary (clinical)}

Organ

dysfunction

free survival
Survival free of organ dysfunction
- Organ dysfunction defined by Paediatric Logistic Organ Dysfunction 2 Score - PELOD-2) [24]

- A PELOD-2 score of $>0$ indicates organ dysfunction

- Censored at 28 days post randomisation

- Assume PELOD-2 is zero at discharge from PICU in survivors

- PELOD-2 calculated using the worst values of individual components each day until discharge

- if a certain variable (such as creatinine) was not measured on a given day it is assumed to be normal [24]

- AKI defined as per KDIGO 2012 criteria using serum creatinine values [19]

- Includes AKI within the first seven days post randomisation if not present on admission. Serum creatinine values measured in the first 7 days post randomisation will be used to assess AKI as per KDIGO 2012 criteria

- Baseline creatinine: closest serum creatinine value prior to randomization, up to 12 hours before randomisation

- Predicted baseline creatinine: for children $<1$ year, the reported predicted creatinine values by Boer et al will be used while for children $>1$ year the below formula will be employed [20].

Mean creatinine $($ micromol/L) $=-2.37330-12.91367$

$* \log _{e}($ age $)+23.93581 *(\text { age })^{1 / 2}[21]$ 
For those with baseline creatinine -

- $\quad$ Baseline creatinine $\geq 1.5$ times predicted baseline creatinine for those with baseline creatinine

For those without baseline creatinine -

- Patients will be assumed to have no AKI on admission

New onset AKI:

For those with baseline creatinine -

- $\quad$ New onset AKI will be according to KIDIGO 2012 criteria using follow-up creatinine values. If no follow-up creatinine available, these patients would be deemed to have no AKI

For those without baseline creatinine -

- $\quad$ Predicted baseline creatinine value will be calculated and assumed to be the baseline value. This value will be compared to follow-up value (where available) as per KDIGO 2012 criteria. Where no follow-up value is available, these patients would be deemed to have no AKI.

Length of stay Length of stay

- Length of stay in PICU from randomisation to discharge

- Length of stay in hospital from randomisation to discharge

Survival

PICU free survival

- Censored at 28 days from the post randomisation

- Patients will be assumed to be alive once discharged from PICU

- For patients who die within 28 days, are discharged to a hospice or for palliative care, this value will be recorded as zero

\section{Secondary safety outcomes, defined as serum electrolyte/metabolite abnormalities present from randomization to 48 hours post randomisation}

\begin{tabular}{|c|c|c|c|}
\hline \multirow[t]{7}{*}{ Adverse event } & Hyperkalemia & 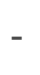 & Serum potassium $>6.2 \mathrm{mmol} / \mathrm{L}$ \\
\hline & Hypokalemia & - & Serum potassium $<2.5 \mathrm{mmol} / \mathrm{L}$ \\
\hline & Hypercalcemia & - & Serum corrected total calcium $>3.1 \mathrm{mmol} / \mathrm{L}$ \\
\hline & Hypocalcemia & - & Serum corrected total calcium $<1.6 \mathrm{mmol} / \mathrm{L}$ \\
\hline & Hypermagnesemia & - & Serum magnesium $>1.4 \mathrm{mmol} / \mathrm{L}$ \\
\hline & Hyponatremia & - & Serum sodium $<125 \mathrm{mmol} / \mathrm{L}$ \\
\hline & Hyperlactatemia & - & Arterial or venous blood gas lactate $>4 \mathrm{mmol} / \mathrm{L}$ \\
\hline & Death in hospital & - & Number of deaths \\
\hline
\end{tabular}


Legend: PICU - Paediatric Intensive Care Unit, AKI - Acute Kidney Injury, KDIGO - Kidney Disease: Improving Global Outcomes, PELOD-2 - Paediatric Logistic Organ Dysfunction 2 Score

\section{Participant timeline $\{13\}$}

The duration of the allocated IVFT treatment will extend until day 28 or one of the following, whichever occurs first:

- discharge from PICU, including transfer to another intensive care facility;

- death;

- withdrawal of consent; or

- occurrence of a major adverse event likely related to the intervention.

Enrolment, interventions and assessments are outlined in figure 1.

\section{Sample size $\{14\}$}

The prevalence of a ${ }^{3} 5 \mathrm{mmol} / \mathrm{L}$ rise in serum chloride level within the first 24 hours in children admitted to the PICU has been reported as 12.5\% [22]. Based on a review of our institutional PICU data, we expect the proportion of children admitted to PICU requiring IVFT that have a ${ }^{3} 5 \mathrm{mmol} / \mathrm{L}$ rise in chloride level to be approximately $20 \%$ by 48 hours. To demonstrate a $10 \%$ absolute reduction in prevalence of this primary outcome (setting statistical thresholds of 0.05 for type I error and $80 \%$ power), we will need a total of 435 patients. To account for $10 \%$ attrition, we will aim to recruit 480 patients. Accounting for ineligible and missed patients, we expect to achieve the recruitment target within an 18-month study period.

\section{Recruitment $\{15\}$}

An automated extraction of data items required for screening will be undertaken daily from the electronic medical records of all PICU admissions to create a list of all eligible patients. The research nurse will screen this list to determine eligible patients. On weekdays, the research nurse will screen all patients admitted to the PICU each morning. During on-call hours and weekends, the admitting registrar or consultant intensivist will screen for eligibility for recruitment. The reasons for ineligibility and for nonparticipation of eligible candidates will be documented by the clinical research nurse in the screening log. Co-enrollment with other studies (with different primary outcomes) will be allowed. Where there are repeat admissions to PICU, patients are eligible for re-randomisation if the readmission occurs after 28 days of the previous randomisation.

\section{Assignment of interventions: allocation}

\section{Sequence generation $\{16 \mathrm{a}\}$}

Online variable block randomisation with block sizes of 3, 6 and 9 and equal 1:1:1 allocation into each of the study interventions NS, PL and CSL will be administered through the online REDCap randomisation 
tool hosted by the University of Queensland.

\section{Concealment mechanism $\{16 \mathrm{~b}\}$}

REDCap online randomisation tool will be employed to implement allocation sequence.

\section{Implementation $\{16 \mathrm{c}\}$}

Automated generation of the allocation sequence will occur in the REDCap trial database. The bedside nurse or the clinician will enroll patients. REDCap online ramdomisation will assign participants to interventions.

\section{Assignment of interventions: Blinding}

\section{Who will be blinded $\{17 \mathrm{a}\}$}

Not applicable. This is an open-label trial.

\section{Procedure for unblinding if needed $\{17 \mathrm{~b}\}$}

Not applicable. There is no blinding as part of interventions in this study.

\section{Data collection and management}

\section{Plans for assessment and collection of outcomes $\{18 \mathrm{a}\}$}

Data collection will be performed using a combination of manual data entry into a REDCap study database $(25,26)$ and automated data extraction from the PICU clinical information system (MetaVisionV 5ä, iMDsoft, Tel Aviv) (Appendix 1). Structured query language (SQL) will be used to automate the extraction of the relevant, routinely collected clinical data values from MetaVision ${ }^{\mathrm{TM}}$. Information on demographics, severity on admission, diagnostic codes, interventions, and length of stay will be extracted through the mandatory data fields which the institution provides to the Australian and New Zealand Paediatric Intensive Care Registry(27). Further detail data collection methods are included in appendix 1 as part of the statistical analysis plan.

\section{Plans to promote participant retention and complete follow-up $\{18 \mathrm{~b}\}$}

Participants will remain in the study until discharge from PICU. Data on protocol violations, eligibility and recruitment rate will be reported. Fluids that are considered as part of the intervention are: any fluid bolus and any IV maintenance. Total parenteral nutrition, enteral feeds and fluids, fluid used in drug dilution, fluid used as intravenous flush and blood products (packed red cells, fresh frozen plasma, cryoprecipitate) will not be considered for protocol violations. Use of $4 \%$ and $20 \%$ albumin will be considered a protocol violation. 
Manual primary source verification will be performed in $100 \%$ of cases for the inclusion criteria and the primary outcome through independent monitoring staff. Extracted data values for random selected $5 \%$ of the study cohort will be manually assessed against the user-interface of MetaVision ${ }^{\mathrm{TM}}$.

\section{Confidentiality $\{27\}$}

Participant confidentiality will be strictly held in trust by the participating investigators, research staff, and the sponsoring institution and their agents. The study protocol, documentation, data and all other information generated will be held in strict confidence. Any data, forms, reports and other records that leave the site will be identified only by a participant identification number (Participant ID, PID) to maintain confidentiality. All records will be stored in a secure online trial database. All computer entry and networking programs will be done using PIDs only. No information concerning the study, or the data will be released to any unauthorized third party, without prior written approval of the sponsoring institution. Clinical information will not be released without written permission of the participant, except as necessary for monitoring by human research ethics committee or regulatory agencies.

\section{Plans for collection, laboratory evaluation and storage of biological specimens for genetic or molecular analysis in this trial/future use $\{33\}$}

Not applicable. No biological samples will be collected for genetic or molecular analysis as part of this trial.

\section{Statistical methods}

\section{Statistical methods for primary and secondary outcomes $\{20 \mathrm{a}\}$}

A per-protocol (PP) analysis will also be undertaken for the primary outcome only including all children who were eligible, randomised, where consent is available, who received the allocated fluid and where both baseline and follow-up chloride measures were obtained.

\section{Interim analyses $\{21 \mathrm{~b}\}$}

A formal interim analysis will be performed after recruitment of 176 children. There are no predetermined stopping rules for this trial.

\section{Methods for additional analyses (e.g. subgroup analyses) \{20b\}}

We will repeat the ITT and PP analyses for the following subgroups for the primary outome:

- Age at PICU admission: $£ 6$ months, $>6$ months to 5 years, $>5$ years to $<16$ years

- Admission type: elective versus non-elective admissions

- Patients who received IV fluids for: $>24$ hours, $\leq 24$ hours 
- Patients who received: $>50 \mathrm{ml} / \mathrm{kg}$ IVFT in the first 48 hours since randomisation, $\leq 50 \mathrm{ml} / \mathrm{kg} \mathrm{IVFT} \mathrm{in}$ the first 48 hours since randomisation

The age subgroup was chosen to delineate the effect of IVFT on neonates and small infants, young children and adolescents. Children who are non-electively admitted to PICU may be sicker and hence, might receive IVFT for longer. Furthermore, they might have more marked metabolic derangement and respond to IVFT differently to fewer sick patients. Hence, we will be analysing the primary outcome in this subgroup separately. As we hypothesise that the increase in chloride level will be proportional to the amount of intravenous fluid received, we will look at specific sub-group of patients who received IVFT for $>24$ hours and those who received $>50 \mathrm{ml} / \mathrm{kg}$ (empirical cutoff) in the first 48 hours since randomisation.

\section{Methods in analysis to handle protocol non-adherence and any statistical methods to handle missing data $\{20 \mathrm{c}\}$}

The proportion of patients who received the allocated fluid will be reported, and the proportion of contamination in $\mathrm{ml} / \mathrm{kg}$ per day for the first 48 hours, and $\mathrm{ml} / \mathrm{kg}$ for the entire admission will be reported. Additionally, the number of patients who had baseline chloride and repeat chloride level measured, number of patients who were administered IVFT for $>24$ hours and who received $>50 \mathrm{ml} / \mathrm{kg}$ (empirical cutoff) in the first 48 hours since randomisation will be reported in total and for each arm. A detailed statistical analysis plan is included in appendix 1.

\section{Plans to give access to the full protocol, participant level-data and statistical code $\{31 \mathrm{c}\}$}

The authors can provide the full protocol. The statistical analysis plan is attached as appendix. The statistical code is also available at https://github.com/kgibbons44/SPLYTPAnalysis/.

\section{Oversight and monitoring}

\section{Composition of the coordinating centre and trial steering committee $\{5 \mathrm{~d}\}$}

SR, AS, LS, MK and KG comprise the steering committee.

\section{Composition of the data monitoring committee, its role and reporting structure $\{21 \mathrm{a}\}$}

While there is not data safety monitoring board for this study, the trial will be overseen by a Trial Steering Committee (TSC), the membership of which will include: the PI, 2 further PIs, senior epidemiologist/statistician and a senior research nurse. The role of the TSC will be to monitor and supervise progress of the trial and review at regular intervals relevant information from other sources.

\section{Adverse event reporting and harms $\{22\}$}

Commonly expected complications related to the underlying disease will not be reported as an adverse event. This may be organ dysfunction such as cardiac or respiratory failure, need for inotropic support or death. Renal dysfunction will be noted separately, as this would be one of the secondary outcomes. 
Potential intervention specific adverse events might be hyperkalemia, hypokalemia, hypercalcemia, hypocalcemia, hypermagnesemia, hyponatremia, hyperlactatemia and death in hospital.

Laboratory biochemistry values will be reviewed every day by the attending clinician and where concerns of an AE exist, this will be discussed with the PI and documented by the clinical research nurse. As these events may be due to the fact that the patient is sick, the attending clinician will be requested to state if in their personal opinion the event potentially could be related to the intervention.

For the purposes of this study the site investigator is responsible for recording all AEs, regardless of their relationship to study intervention, for the period from randomisation until discharge from hospital. Conditions that are present at screening and do not deteriorate will not be considered AEs. AEs will be reported to the $\mathrm{PI}$ as soon as possible. The $\mathrm{PI}$ along with the rest of the research team will review all AEs and determine relatedness and severity. Complications and side effects will be reported using the existing hospital internal reporting structures and databases. The PI will then report back to the patients where appropriate and the ethics committee.

\section{Frequency and plans for auditing trial conduct $\{23\}$}

While ongoing regular trial conduct and data monitoring is in place, specific timepoints, frequency and procedures for formal audits are not planned.

\section{Plans for communicating important protocol amendments to relevant parties (e.g. trial participants, ethical committees) $\{25\}$}

All protocol amendments will be approved by the HREC. The ANZPICR application will also be amended accordingly to mirror these changes.

\section{Dissemination plans $\{31$ a $\}$}

Given the lead role of the investigators in paediatric intensive care; their role in regional and state-wide guideline development processes; their involvement in professional colleges and at key educational conferences it is likely that the findings will have nationwide impact across Australia and New Zealand.

Publication in high impact peer-reviewed journals will be sought and presentation at national and international conferences is anticipated. Novel and modern information dissemination strategies will also be used including social media, podcast presentations and Free Open Access Medical education (FOAM) resources to generate discussion and disseminate the outcomes of the study.

\section{Discussion}

IVFT is the most common intervention that critically ill children are exposed to. Our trial will be one of the largest randomised controlled trial investigating intravenous fluid management in critically ill children. In 
addition, given how commonly IVFT is used outside the PICU, trial findings may be directly relevant to children admitted to every hospital.

Our trial is designed as a highly pragmatic trial. Data collection leverages off automated data extraction from integrated Electronic Medical Record and MetaVision ${ }^{\mathrm{TM}}$ systems. While making it a robust trial, this methodology has transformed it into a relatively low-cost large randomised control trial. The trial design has the potential to inform future pragmatic trials using electronic health record information.

\section{Trial status}

The current protocol is the version 7, dated 29/07/2020. Recruitment commenced on $12^{\text {th }}$ November 2019. The tentative date for completion of recruitment is $1^{\text {st }}$ May 2021.

\section{Abbreviations}

\begin{tabular}{ll} 
Abbreviation & Term \\
\hline AKI & Acute Kidney Injury \\
\hline ATSI & Aboriginal and Torres Strait Islander \\
\hline Cl & Confidence Interval \\
\hline CSL & Compound Sodium Lactate \\
\hline ED & Emergency Department \\
\hline FFP & Fresh Frozen Plasma \\
\hline HFNC & High Flow Nasal Cannula \\
\hline ITT & Intention-to-Treat \\
\hline IVFT & Intravenous Fluid Therapy \\
\hline KDIGO & Kidney Disease: Improving Global Outcomes \\
\hline NS & 0.9\% Sodium Chloride \\
\hline PELOD-2 & Paediatric Logistic Organ Dysfunction 2 Score \\
\hline PICU & Paediatric Intensive Care Unit \\
\hline PL & Plasma-Lyte 148 \\
\hline PP & Per Protocol \\
\hline RBC & Packed Red Blood Cells \\
\hline RCT & Randomised Controlled Trial \\
\hline SQL & Structured Query Language \\
\hline
\end{tabular}




\section{Declarations}

\section{Acknowledgements}

We acknowledge the PICU medical and nursing team of Queensland Children's Hospital who actively support the recruitment of patients into this study.

\section{Authors' contributions \{31b\}}

SR developed the concept, sourced funding, administers the project, will write up results and report adverse events. MK administers the project, screen and collect data. RLM created the trial database and will enable automated data extraction. PT and AM advised on protocol design and study concept. AS and KG advised on protocol design, the conduct of the trial and statistical analyses. KG will be supervising analyses. LJS supervised protocol design, study setup, and advises on conduct of the trial, data collection and statistical analyses.

\section{Funding $\{4\}$}

The study was supported by a grant from the Study, Education and Research Trust Account funding scheme 2019, Children's Health Queensland, Brisbane, Australia. The funding sources had no involvement in study design, analyses, nor interpretation of the results.

\section{Availability of data and materials $\{29\}$}

The research team will have access to the trial dataset. Anonymised data could be shared with other investigators for further exploratory analyses after formal approval from the research committee and sponsoring institution.

\section{Ethics approval and consent to participate $\{24\}$}

The study protocol has been reviewed and approved by ethics committee in Australia (Children's Health Queensland Human Research Ethics Committee, HREC/19/QCHQ/53177).

\section{Consent for publication $\{32\}$}

Prospective informed consent will be sought from parents or carers on consent form that has been reviewed by the HREC. Authors can provide a model consent form on request.

\section{Competing interests $\{28\}$}

All authors have no conflicts of interest to declare.

\section{Authors' information (optional)}

Information about author contribution presented earlier. 


\section{References}

1. Finfer S, Myburgh J, Bellomo R. Intravenous fluid therapy in critically ill adults. Nature Reviews Nephrology. 2018;14(9):541-57.

2. Alobaidi R, Morgan C, Basu RK, Stenson E, Featherstone R, Majumdar SR, et al. Association between fluid balance and outcomes in critically ill children: A systematic review and meta-analysis. JAMA Pediatrics. 2018;172(3):257-68.

3. Awad S, Allison SP, Lobo DN. The history of $0.9 \%$ saline. Clinical Nutrition. 2008;27(2):179-88.

4. Huber JL, Berger S, Löllgen RM. Balanced Electrolyte Solutions or Normal Saline? Resuscitative Fluid Administration Practice in Swiss Pediatric Acute Care. Pediatric Emergency Care. 2019;00(00):1-

5. Varrier M, Ostermann M. Fluid Composition and Clinical Effects. Crit Care Clin. 2015;31(4):823-37.

6. Feld LG, Neuspiel DR, Foster BA, Leu MG, Garber MD, Austin K, et al. Clinical Practice Guideline: Maintenance Intravenous Fluids in Children. Pediatrics. 2018;142(6).

7. Severs D, Hoorn EJ, Rookmaaker MB. A critical appraisal of intravenous fluids: From the physiological basis to clinical evidence. Nephrology Dialysis Transplantation. 2015;30(2):178-87.

8. Orbegozo Cortés D, Rayo Bonor A, Vincent JL. Isotonic crystalloid solutions: A structured review of the literature. 2014. p. 968-81.

9. Morgan TJ. The meaning of acid-base abnormalities in the intensive care unit: part III - effects of fluid administration. Crit Care. 2005;9(2):204-11.

10. Bulfon AF, Alomani HL, Anton N, Comrie BT, Rochwerg B, Stef SA, et al. Intravenous Fluid Prescription Practices in Critically III Children: A Shift in Focus from Natremia to Chloremia? J Pediatr Intensive Care. 2019;8(4):218-25.

11. Self WH, Semler MW, Wanderer JP, Wang L, Byrne DW, Collins SP, et al. Balanced Crystalloids versus Saline in Noncritically III Adults. New England Journal of Medicine. 2018;378(9):819-28.

12. Semler MW, Self WH, Wanderer JP, Ehrenfeld JM, Wang L, Byrne DW, et al. Balanced Crystalloids versus Saline in Critically III Adults. The New England journal of medicine. 2018;378(9):829-39.

13. Young P, Bailey M, Beasley R, Henderson S, Mackle D, McArthur C, et al. Effect of a Buffered Crystalloid Solution vs Saline on Acute Kidney Injury Among Patients in the Intensive Care Unit: The SPLIT Randomized Clinical Trial. Jama. 2015;314(16):1701-10.

14. Weiss SL, Keele L, Balamuth F, Vendetti N, Ross R, Fitzgerald JC, et al. Crystalloid Fluid Choice and Clinical Outcomes in Pediatric Sepsis: A Matched Retrospective Cohort Study. The Journal of pediatrics. 2017;182(0):304-10.e10.

15. Kartha GB, Rameshkumar R, Mahadevan S. Randomized Double-blind Trial of Ringer Lactate Versus Normal Saline in Pediatric Acute Severe Diarrheal Dehydration. Journal of Pediatric Gastroenterology and Nutrition. 2017;65(6):621-6.

16. Allen $\mathrm{CH}$, Goldman RD, Bhatt S, Simon HK, Gorelick MH, Spandorfer PR, et al. A randomized trial of Plasma-Lyte A and $0.9 \%$ sodium chloride in acute pediatric gastroenteritis. BMC Pediatrics. 2016;16(1):1-9. 
17. Lima MF, Neville IS, Cavalheiro S, Bourguignon DC, Pelosi P, Malbouisson LMS. Balanced Crystalloids Versus Saline for Perioperative Intravenous Fluid Administration in Children Undergoing Neurosurgery: A Randomized Clinical Trial. Journal of Neurosurgical Anesthesiology. 2019;31(1):305.

18. Balamuth F, Kittick M, McBride P, Woodford AL, Vestal N, Casper TC, et al. Pragmatic Pediatric Trial of Balanced Versus Normal Saline Fluid in Sepsis: The PRoMPT BOLUS Randomized Controlled Trial Pilot Feasibility Study. Academic Emergency Medicine. 2019;26(12):1346-56.

19. Kellum JA, Lameire N. Diagnosis, evaluation, and management of acute kidney injury: a KDIGO summary (Part 1). Critical care (London, England). 2013;17(1):204-.

20. Boer DP, De Rijke YB, Hop WC, Cransberg K, Dorresteijn EM. Reference values for serum creatinine in children younger than 1 year of age. Pediatric Nephrology. 2010;25(10):2107-13.

21. Ceriotti F, Boyd JC, Klein G, Henny J, Queraltó J, Kairisto V, et al. Reference intervals for serum creatinine concentrations: Assessment of available data for global application. Clinical Chemistry. 2008;54(3):559-66.

22. Barhight MF, Brinton J, Stidham T, Soranno DE, Faubel S, Griffin BR, et al. Increase in chloride from baseline is independently associated with mortality in critically ill children. Intensive Care Medicine. 2018;44(12):2183-91.

23. Levin M, Cunnington AJ, Wilson C, Nadel S, Lang HJ, Ninis N, et al. Effects of saline or albumin fluid bolus in resuscitation: evidence from re-analysis of the FEAST trial. The Lancet Respiratory Medicine. 2019;7(7):581-93.

24. Leteurtre S, Duhamel A, Salleron J, Grandbastien B, Lacroix J, Leclerc F. PELOD-2: an update of the PEdiatric logistic organ dysfunction score. Critical care medicine. 2013;41(7):1761-73.

25. Harris PA, Taylor R, Thielke R, Payne J, Gonzalez N, Conde JG. Research electronic data capture (REDCap)-a metadata-driven methodology and workflow process for providing translational research informatics support. Journal of biomedical informatics. 2009;42(2):377-81.

26. Harris PA, Taylor R, Minor BL, Elliott V, Fernandez M, O'Neal L, et al. The REDCap consortium: Building an international community of software platform partners. J Biomed Inform. 2019;95:103208.

27. Schlapbach LJ, Straney L, Alexander J, MacLaren G, Festa M, Schibler A, et al. Mortality related to invasive infections, sepsis, and septic shock in critically ill children in Australia and New Zealand, 2002-13: a multicentre retrospective cohort study. Lancet Infect Dis. 2015;15(1):46-54.

\section{Figures}




\begin{tabular}{|c|c|c|c|c|}
\hline & \multicolumn{4}{|c|}{ STUDY PERIOD } \\
\hline & Enrolment & Allocation & Post-allocation & Close-out \\
\hline TIMEPOINT** & $-t_{1}$ & 0 & $\begin{array}{l}\text { For the duration of } \\
\text { intravenous fluid therapy } \\
\text { whilst admitted to PICU }\end{array}$ & $\begin{array}{l}\text { Discharge } \\
\text { from PICU }\end{array}$ \\
\hline \multicolumn{5}{|l|}{ ENROLMENT: } \\
\hline Eligibility screen & $\mathrm{x}$ & & & \\
\hline Informed consent & $\mathrm{x}$ & & & \\
\hline Allocation & & $\mathrm{x}$ & & \\
\hline \multicolumn{5}{|l|}{ INTERVENTIONS: } \\
\hline $\begin{array}{l}\text { Start of randomised } \\
\text { intravenous fluid therapy }\end{array}$ & & & $\longmapsto$ & \\
\hline $\begin{array}{l}\text { Daily blood gases } \\
\text { until discharge from PICU }\end{array}$ & & & $\mathrm{x}$ & \\
\hline \multicolumn{5}{|l|}{ ASSESSMENTS: } \\
\hline \multicolumn{5}{|l|}{ List of Baseline variables } \\
\hline $\begin{array}{l}\text { 1. Screening variables } \\
\text { 2. Serum chloride level before } \\
\text { randomisation } \\
\text { 3. Biochemical values before } \\
\text { randomisation }\end{array}$ & $x$ & $x$ & & \\
\hline \multicolumn{5}{|l|}{$\begin{array}{l}\text { 4. Date of admission } \\
\text { Demographics } \\
\text { 5. Presenting diagnosis } \\
\text { 6. Intravenous fluid } \\
\text { administered before admission } \\
\text { to PICU } \\
\text { 7. Biochemical values before } \\
\text { admission to PICU }\end{array}$} \\
\hline \multicolumn{5}{|l|}{ List of Outcome Variables } \\
\hline $\begin{array}{l}\text { 1. Date of Discharge } \\
\text { 2. PICU length of stay } \\
\text { 3. Hospital length of stay } \\
\text { 4. Death }\end{array}$ & & & & $x$ \\
\hline \multicolumn{5}{|l|}{ List of other data variables } \\
\hline $\begin{array}{l}\text { 1. Physiological variables } \\
\text { 2. Maximum chloride in PICU } \\
\text { 3. Pediatric Logistic Organ } \\
\text { Dysfunction Score variables } \\
\text { 4. Intravenous fluids } \\
\text { administered from PICU } \\
\text { admission to discharge } \\
\text { 5. Organ support } \\
\text { 6. Parameters for assessment } \\
\text { of Acute Kidney Injury } \\
\text { 7. Protocol deviations } \\
\text { 8. Adverse events }\end{array}$ & & & $x$ & $\mathrm{x}$ \\
\hline
\end{tabular}

\section{Figure 1}

The schedule of enrolment, interventions, and assessments.

\section{Supplementary Files}

This is a list of supplementary files associated with this preprint. Click to download. 
- 20201126Appendix1SPLYTPProtocolpaperSAP.docx 